\title{
Telemedicine in the follow-up of diabetic patients: Utility and effectiveness studied in lockdown
}

\author{
Meryem DRISSI OUDGHIRI*, Saloua ELAMARI*, Imane MOTAIB, Soukaina LAIDI, Asmaa CHADLI. \\ *Both authors have contributed equally to this article.
}

Department of endocrinology, diabetology, metabolic diseases and nutrition.
University Mohamed VI of health sciences, 82403, Casablanca, Morocco.

DOI: 10.29322/IJSRP.11.12.2021.p12002

http://dx.doi.org/10.29322/IJSRP.11.12.2021.p12002

\begin{abstract}
Introduction: Telemedicine has found renewed interest during the lockdown period caused by the COVID 19 pandemic. The aim of this study is to compare variations in the metabolic parameters of diabetic patients followed in consultation and those followed in teleconsultation during the confinement period.

Patients and Methods: This prospective study took place between April and May 2020 and included diabetic patients followed in their consultation, appointment during the confinement period. We excluded patients with less than 2 consultations during the year 2019, and patients who did not attend the consultation after the confinement period came to an end. We compared demographic and metabolic characteristics, treatment compliance, stress level, and change in caloric intake during the confinement period.

Results: We collected 65 patients. Thirty-one were follow-ups at their consultations and 34 were Telemedicine follow-ups. The median age was 62 and 55.5 years, respectively. The sex ratio $\mathrm{H}$ $/ \mathrm{F}$ was 1 and 0.9 respectively and the WT2 was $87.3 \%$ and $88 \%$ respectively. The median BMI before confinement was 29.5 and $28 \mathrm{~kg} / \mathrm{m}^{2}$ respectively. There was no significant difference between the 2 groups concerning the increase in caloric intake and the post-confinement variation in weight and HBA1C. Assessment of stress levels and treatment compliance showed significantly higher stress levels and significantly better treatment compliance in the patients followed in consultation.

Discussion and Conclusion: Teleconsultation has allowed us to follow more diabetic patients during the confinement period. With an efficiency comparable to consultation, Telemedicine allows access to regular follow-ups for a large population and facilitates patient/caregiver exchange. Regulations are needed to legalize the exchange, protect data and enhance the value of teleconsultation as an act of care.
\end{abstract}

Index terms: Telemedicine, diabetes, lockdown.

\section{INTRODUCTION:}

Since December 2019, a new coronavirus has been identified in Wuhan and was named severe acute respiratory syndrome coronavirus 2 (SARS-CoV-2) [1].

The World Health Organization (WHO) declared on March 11, 2020, the 2019 coronavirus disease as a global pandemic [2].

This publication is licensed under Creative Commons Attribution CC BY.

http://dx.doi.org/10.29322/IJSRP.11.12.2021.p12002
Many countries have practiced a lock-down to limit the spread of the COVID-19 infection [3]. Morocco decided to establish containment for 94 days, starting from March 20, 2020.

This confinement had repercussions in patients with diabetes; which is a chronic metabolic condition characterized with hyperglycemia and associated with metabolic complications [4]; like decreased or even cessation of physical activity, increased caloric intake, but also restricted access to care due to hospital overcrowding and restricted discharges. Any or all of these factors can lead to acute or chronic glycemic imbalance and aggravation of diabetes-related co-morbidities.

Telemedicine can help patients connect with their doctors while staying at home, away from hospitals, as travel to hospitals or other health care facilities may increase the risk of exposure to coronavirus infection [5]. As a result, Telemedicine may be useful in managing patients with chronic diseases, such as diabetes, during this pandemic containment period.

The objective of this work is to study the effectiveness of telemedicine follow-up by comparing the anthropometric, metabolic, and biological parameters of diabetic patients followed in consultation and those followed in teleconsultation during the confinement period.

\section{METHODOLOGY:}

\section{Study design:}

This is a prospective study between April and May 2020 including diabetic patients followed at the consultation with an appointment during the confinement period. We excluded patients with less than 2 consultations during the year 2019, and patients who did not attend the consultation after the confinement was lifted. We compared demographic, metabolic characteristics, treatment compliance, stress level, and change in caloric intake during the confinement period.

\section{Definitions}

We considered the weight and height of patients taken during the consultation before and after confinement. They were measured using standard equipment. Weight was measured to the nearest 0.5 $\mathrm{kg}[6]$

Treatment adherence was assessed using a self-administered questionnaire derived from the Morisky-Green Medication Adherence Questionnaire (MAQR) [7] 
Caloric intake was assessed by a 24-hour recall.

The stress level was assessed by the stress perception scale [8]. Glycemic control was assessed by the HbA1c study treated by the HPLC technic

Acute complications of diabetes are blood glucose levels below $0.7 \mathrm{~g} / \mathrm{l}$ or above $4 \mathrm{~g} / \mathrm{l}$ or the occurrence of diabetic ketosis.

Teleconsultation:

We have considered as teleconsultation any consultation carried out via the Visiomedica platform.

\section{Data Collection}

We extracted the demographic information, anthropometric parameters and initial biological parameters of all diabetic patients followed in the consultation at Sheikh Khalifa Hospital during the period of confinement via conventional consultation, or teleconsultation through the Visiomedica platform and collected the same information in conventional consultation after confinement.

4. Measuring Results

Data from diabetic patients followed within our structure was compared with data from the same patients in post-confinement to study any differences in the demographic characteristics, anthropometric parameters and biological parameters and the impact of remote monitoring by teleconsultation on these parameters.

We described the age, co-morbidities, known diabetes complications and treatment of patients.

Caloric intake is considered increased if the pre-confinement caloric intake grows by more than $30 \%$.

We studied the stress level using the stress level scale.

*Score below 21: This is a person who knows how to manage his stress, how to adapt and for whom there are always solutions.

*Score between 21 and 26: This is a person who generally knows how to cope with stress but is currently experiencing emotional turmoil.

*Score above 27: Life is a constant threat to this person.

Adherence to therapy was considered good for those scoring 8 or higher, average for those scoring 6 or 7 , and low for those scoring less than 6. [7]

We collected the number of acute complications based on patients' self-monitoring logs.

We compared weight, body mass index and HBA1C before and after lock-down.

\section{Statistical Analysis}

The data was analyzed using SPSS (V.20.0). A value of $\mathrm{p}<0.05$ was considered statistically significant. The study of the normality of distribution of continuous variables was performed by the Kolmogorov-Smirnov test. An independent sample t-test was applied to analyze normally distributed data and the MannWhitney test was applied to analyze normally undistributed data. A paired sample t-test was used to compare quantitative variables before and after containment. The $\chi 2$ test and the Fisher test were applied to examine categorical data.

\section{RESULTS:}

\section{Patient Demographics:}

We collected 65 patients.

This publication is licensed under Creative Commons Attribution CC BY http://dx.doi.org/10.29322/IJSRP.11.12.2021.p12002
Thirty-one of them were followed up at the conventional consultation, i.e., $48 \%$ (group 1) and 34 were followed up by telemedicine, i.e., $52 \%$ (group 2).

The median age was 62 and 55.5 years, respectively $(\mathrm{p}=0.77)$.

The sex ratio of $\mathrm{M} / \mathrm{F}$ was 1 and 0.9 , respectively $(\mathrm{p}=0.60)$ (Table $1)$.

2. Background:

Type 2 diabetes accounted for $87.3 \%$ and $88 \%$ respectively in each group $(\mathrm{p}=0.43)$.

Patients on insulin therapy accounted for $64.5 \%$ and $70.5 \%$ respectively in each group $(\mathrm{p}=0.09)$.

Patients on oral anti-diabetic drugs represented $35.48 \%$ in the conventional consultation group and $26.47 \%$ in the teleconsultation group $(\mathrm{p}=0.49)$.

In the conventional consultation group, patients had more cardiovascular risk factors $(64.51 \%$ versus $32.35 \%)(p=0.05)$ and more chronic complications of diabetes than in the teleconsultation group $(51.6 \%$ versus $17.64 \%)(p=0.04)($ Table 1$)$.

\section{Metabolic parameters of patients before containment:}

Mean weight was $79.86(15.03)$ in the conventional consultation group and 76.58 (12.93) in the teleconsultation group $(\mathrm{p}=0.47)$.

The median pre-containment BMI was $29.5 \mathrm{~kg} / \mathrm{m}^{2}$ in the conventional consultation group and $28 \mathrm{~kg} / \mathrm{m}^{2}$ in the teleconsultation group $(\mathrm{p}=0.31)$.

The average hbalc is $7.9 \%$ in the conventional consultation group versus $7.79 \%$ in the teleconsultation group $(\mathrm{p}=0.83)$ (Table 1$)$.

4. Analysis of patients' experiences during confinement:

*Assessment of stress level:

The high-stress level in $25 \%$ of the conventional consultation group and $9 \%$ of the patients in the teleconsultation group $(\mathrm{p}<0.05)$ (Table 2)

*Evaluation of therapeutic adherence:

In the conventional consultation group, $87.09 \%$ of patients were poorly compliant versus $29.4 \%$ in the teleconsultation group $(\mathrm{p}<0.05)$ (Table 2).

*Occurrence of acute complications:

Patients followed in teleconsultation had more hypoglycemia $(14.7 \%)$ than those followed in conventional consultation $(9.6 \%)$ $(\mathrm{p}=0.42)$, while episodes of hyperglycemia and ketosis were quite similar in both groups $(\mathrm{p}=056 ; \mathrm{p}=0.42$ respectively) (Table 2$)$

5. Analysis of metabolic parameters in post-lockdown

* Metabolic parameters:

Weight gain occurred in $64.5 \%$ of patients in the conventional consultation group versus $58.8 \%$ in the teleconsultation group $(\mathrm{p}=0.41)$. The change in mean weight was +0.5 in the conventional consultation group compared to +0.44 in the teleconsultation group $(\mathrm{p}=0.58)$.

In the conventional consultation group, $49.90 \%$ of patients had an hbalc greater than $8 \%$ in post-confinement, versus $41.20 \%$ of patients in the teleconsultation group $(\mathrm{p}=0.32)$. The change in mean hba1c was $+0.19 \%$ in the conventional consultation group versus an increase of $+0.12 \%$ in the teleconsultation group $(\mathrm{p}=0.02)$.

There was also an increase in caloric intake of $29.03 \%$ in the conventional consultation group, versus $29.41 \%$ in the teleconsultation group $(\mathrm{p}=0.58)$. 
Table 1: Demographic analysis, history and metabolic parameters of patients before lockdown

\begin{tabular}{|c|c|c|c|c|}
\hline & Total $(n=65)$ & $\begin{array}{c}\text { Conventional } \\
\text { consultation } \\
(n=31)\end{array}$ & $\begin{array}{l}\text { Teleconsult } \\
(n=34)\end{array}$ & P-value \\
\hline \multicolumn{5}{|l|}{ Demographics } \\
\hline Age, median (IQR), years & $60(52-66)$ & $62(55-66.5)$ & $55.5(47-64)$ & 0.77 \\
\hline Sex ratio H/F & 0.95 & 1 & 0.9 & 0.6 \\
\hline \multicolumn{5}{|l|}{ Medical history } \\
\hline Type 2 diabetes mellitus, $\mathrm{n}(\%)$ & $56(87.65)$ & $27(87.3)$ & $29(88)$ & 0.43 \\
\hline Oral antidiabetic treatment $\mathrm{n}(\%)$ & $20(30.76)$ & $11(35.48)$ & $9(26.47)$ & 0.49 \\
\hline Insulin treatment n (\%) & $44(67.69)$ & $20(64.51)$ & $24(70.58)$ & 0.09 \\
\hline Other vascular risks $\mathrm{n}(\%)$ & $31(47.69)$ & $20(64.51)$ & $11(32.35)$ & 0.05 \\
\hline Chronical complications of diabetes n (\% & $22(33.8)$ & $16(51.6)$ & $6(17.64)$ & 0.04 \\
\hline \multicolumn{5}{|l|}{ Metabolic parameters before lockdown } \\
\hline Weight medium (SD), \% & $78.12(13.94)$ & $79.86(15.03)$ & $76.58(12.93)$ & 0.47 \\
\hline BMI median (IQR), $\mathrm{kg} / \mathrm{m}^{2}$ & $28.5(27-31.5)$ & $29.5(28-32)$ & $28(25-30)$ & 0.31 \\
\hline A1c medium (SD), \% & $7.84(1.08)$ & $7.9(1.09)$ & $7.79(1.09)$ & 0.83 \\
\hline
\end{tabular}

Table 2 : Parameters analyzed during lockdown and variation of metabolic parameters in post-lockdown

\begin{tabular}{|c|c|c|c|c|}
\hline & Total $(n=65)$ & $\begin{array}{l}\text { Conventionnal } \\
\text { consultation } \\
(n=31)\end{array}$ & $\begin{array}{l}\text { Teleconsult } \\
\qquad(n=34)\end{array}$ & P-value \\
\hline \multicolumn{5}{|c|}{ Analysis of patients' experiences during confinement } \\
\hline High-stress level n (\%) & $34(51.50)$ & $25(80.6)$ & $9(26.47)$ & $<0.05$ \\
\hline Poor adherence to treatment $\mathrm{n}(\%)$ & $37(56.92)$ & 27 (87.09) & $10(29.40)$ & $<0.05$ \\
\hline Diabetes complications n,(\%) & $22(33.84)$ & $16(51.60)$ & $6(17.64)$ & 0.04 \\
\hline hypoglycemia n,(\%) & $8(12.30)$ & $3(9.60)$ & $5(14.70)$ & 0.42 \\
\hline hyperglycemia $n,(\%)$ & $2(3.07)$ & $1(3.20)$ & $1(2.94)$ & 0.56 \\
\hline Diabetic ketosis $n,(\%)$ & $3(4.51)$ & $1(3.20)$ & $2(5.80)$ & 0.42 \\
\hline \multicolumn{5}{|c|}{ Variation of metabolic parameters after lockdown } \\
\hline Weight gain $\mathrm{n}(\%)$ & $40(61.50)$ & $20(64.50)$ & $20(58.80)$ & 0.41 \\
\hline Weight change, medium (SD) & $+0.47(2.43)$ & $+0.5(2.50)$ & $0.44(2.40)$ & 0,58 \\
\hline $\mathrm{A} 1 \mathrm{c}>8 \%, \mathrm{n}(\%)$ & $29(45.30)$ & $15(49.90)$ & $14(41.20)$ & 0.32 \\
\hline A1c variation $\%$, medium(SD) & $+0.15(1.07)$ & $+0.19(1.30)$ & $+0.12(0.70)$ & 0.02 \\
\hline Increased calory intake n (\%) & $19(29.23)$ & $9(29.03)$ & $10(29.41)$ & 0.58 \\
\hline
\end{tabular}

\section{DISCUSSION}

The Coronavirus 2019 (COVID-19) pandemic is an epidemiological event that marks medicine and its actors [9] and lock-down is a very important step in limiting the spread of CoV2 SARS infection [10].

The contribution of telemonitoring for chronic conditions such as diabetes, the use of platforms for transmitting patient data and providing advice, had been booming for people with This publication is licensed under Creative Commons Attribution CC BY. http://dx.doi.org/10.29322/IJSRP.11.12.2021.p12002 diabetes for some years [11], so during this lockdown period, telemedicine is a useful tool for managing patients with diabetes [12].

This situation hindered access to health care, limited face-toface visits in hospitals and threatened the follow-up of diabetic patients during lockdown. To overcome this situation, telemedicine appeared as a feasible and structured alternative to diabetic patients face to face follow-up in the context of the COVID-19 pandemic [13] 
In our study, we compared diabetic patients followed in conventional consultation (group 1) and patients followed by teleconsultation (group 2), considering demographic and metabolic characteristics, treatment adherence, stress level and variation in caloric intake during confinement.

Patients followed in conventional consultation have more cardiovascular risk factors and more chronic degenerative complications of diabetes and have significantly higher stress levels and poor adherence to therapy compared to those followed by teleconsultation. This may explain the increase in hbalc, which was greater in the conventional consultation group (group 1).

A New York meta-analysis by Huey Lee and colleagues of all randomized controlled trials that evaluated the effect of telemedicine follow-up on glycemic control in type 2 diabetes found a decrease in $\mathrm{HbA} 1 \mathrm{c}$ in the telemedicine group by $0.77 \%$, compared to the control group. Of all the different types of telemedicine interventions tested in the trials, tele-education interventions appeared to be the most effective $(-0.71 \% \mathrm{HbA} 1 \mathrm{c}$; $\mathrm{p}<0.05)$. The other types of interventions (telemonitoring: simple monitoring, teleconsultation: risk stratification for referral, tele management: therapeutic decision) were

associated with a decrease in HbA1c of approximately 0.30 $0.40 \%$, but none were significant [14].

A Chinese meta-analysis comparing two similar groups showed a $0.37 \%$ ( $<<0.001)$ reduction in HbA1c in the telemedicine group compared to the control group [15].

In a Cochrane study by Flodgren and colleagues, a reduction in HbA1c of $0.31 \%$. ( $p<0.001)$ was observed in patients monitored by telemedicine compared to the control group [16]. In a Strasbourg study conducted during the pandemic, an overall trend of improvement in HbA1c levels was demonstrated, which was more pronounced in the group followed by teleconsultation and particularly in the hospital [17].

There was no significant difference between the two groups in terms of weight change, increased caloric intake and the occurrence of acute complications.

This supports the value of teleconsultation in the follow-up of diabetic patients.

Since the 1990s, numerous American and European studies on the management of diabetes via telemedicine have shown that telemonitoring of diabetes results in better glycemic control, an improvement in biological markers( in this case glycated hemoglobin) good adherence of patients to therapeutic and lifestyle changes, better control of cardiovascular risk factors (hypertension, dyslipidemia, smoking, overweight and obesity), improved quality of life for patients and increased patient responsibility for their disease with good adherence and compliance [18].

The data for each patient should be confidential and stored on the platform level used. Patient consent, whether implicit (i.e., the patient initiated the consultation themselves) or explicit (i.e., someone else initiated the consultation, either the caregiver or the health care worker), must be obtained. The patient's medical record must be kept with the laboratory and radiology reports and the prescription. If the telemedicine consultation is not considered adequate for the clinical This publication is licensed under Creative Commons Attribution CC BY. http://dx.doi.org/10.29322/IJSRP.11.12.2021.p12002 assessment, the patient should be called for consultation at an appropriate time and place to avoid the risk of coronavirus infection [19].

At the end, maintaining telehealth as an option post-lockdown has the potential to increase timely and safe access to primary health care for many patients [20].

\section{CONCLUSION}

Telemedicine is a useful tool for managing patients of diabetes during this lockdown period. However, there is limited data and further research is required. Access to a treating physician must be facilitated to ensure personalized follow-up, especially for chronic conditions such as diabetes, but also to prevent degenerative complications. Our study showed that follow-up by teleconsultation is effective with results comparable to those of patients followed by conventional consultation in a confined space. Telemedicine would allow access to regular follow-up for a large population and would facilitate patient/caregiver exchange.

Several aspects of teleconsultation will, however, need to be clarified. First, it should be valued as an act of care, we have to guard eligible patient profiles, which will require an adaptation in terms of equipment and computer training for patients and caregivers, mainly concerning the security of transmitted data and their confidentiality.

\section{BIBLIOGRAPHY:}

[1] Zhou F, Ting Y, Du R, et al.: Clinical course and risk factors for mortality of adult in patients with COVID-19 in Wuhan, China: a retrospective cohort study. Lancet. 2020, 395:10541062. 10.1016/S0140-6736(20)30566-3

[2] World Health Organization. Rolling updates on coronavirus disease (COVID-19); 2020. Available from: https://www.who.int/emergencies/diseases/novel-coronavirus2019/events-as-they-happen. Accessed October 22, 2020

[3] COVID-19: Prevention and control measures in community; Rahmet GÜNER, İmran HASANOĞLU, Firdevs AKTAŞ ; Turk J Med Sci. 2020; 50(3): 571-577.2020 Apr 21.

doi: 10.3906/sag-2004-146

[4] Diagnosis and Classification of Diabetes Mellitus; Diabetes Care. 2009 Jan; 32(Suppl 1): S62-S67. doi: 10.2337/dc09-S062 [5] COVID-19 and diabetes : possible role of polymorphism and rise of telemedicine ; Shomoita Sayed ; Department of Mathematics and Natural Science, Brac University, 66 Mohakhali, Dhaka-1212, Bangladesh ; Primary care diabetes PCDE

[6] Body size, silhouette trajectory and the risk of breast cancer in a Moroccan case-control study ; Mohamed Khalis , Laure Dossus , Sabina Rinaldi , Carine Biessy , Aurélie Moskal, Hafida Charaka , Emmanuel Fort, Mathilde His , Nawfel Mellas, Chakib Nejjari, Barbara Charbotel, Amr S. Soliman , Isabelle Romieu, Véronique Chajès , Marc J. Gunter ,Inge Huybrechts, Karima El Rhazi

[7] L'adhésion au traitement médicamenteux, concepts et moyens pour la maintenir ou l'améliorer. Cahier de FC de L'actualité pharmaceutique octobre 2011 
ISSN 2250-3153

[8]Collange J, Bellinghausen L, Chappé J, Saunder L, Albert E. Stress perçu : à partir de quel seuil devient-il un facteur de risque pour les troubles anxiodépressifs ? Archives des Maladies Professionnelles et de l'Environnement. 1 févr 2013;74(1):7 15 .

[9] COVID-19 and diabetes mellitus: from pathophysiology to clinical management ; Soo Lim , Jae Hyun Bae, Hyuk-Sang Kwon Michael A Nauck ; Nat Rev Endocrinol 2021 Jan;17(1):11-30 ; doi: 10.1038/s41574-020-00435-4.

[10] Halimi.S, Médecine des Maladies Métaboliques, Volume 14, Issue 3, May 2020, Pages 191-193

[11] Mise au point sur les projets de recherche dans le domaine de la télémédecine dans le diabète, avec un focus sur les projets de télésurveillance 2.0, Médecine des Maladies Métaboliques,Volume 13, Issue 1, February 2019, Pages 75-87 [12] Telemedicine for diabetes care in India during COVID19 pandemic and national lockdown period: Guidelines for physicians ; AmertaGhosh,RiteshGupta , AnoopMisra

[13] Diabetes During the Fasting Month of Ramadan: Is Telemedicine as Efficient as Conventional Follow-Up? Results from a Moroccan Comparative Study

Imane Motaib, Saloua Elamari, Mohamed Khalis , Meryem Drissi Oudghiri , Soukaina Laidi, Asma Chadli ; Diabetes, Metabolic Syndrome and Obesity: Targets and Therapy

[14] Shaun Wen Huey Lee, Carina Ka Yee Chan, Siew Siang Chua\& Nathorn, Chaiyakunapruk; Comparative effectiveness of telemedicine strategies on type 2 diabetes management: A systematic review and network meta-analysis. Scientific reports. Published 04 october 2017

[15] Zhai Y-K, Zhu W-J, Cai Y-L, Sun D-X, Zhao J. Clinicaland cost-effectiveness of telemedicine in type 2 diabetes mellitus: a systematic review and meta-analysis. Medicine (Baltimore). 2014;93(28):e312.
[16]Timpel P, Oswald S, Schwarz PEH, Harst L. Mapping the evidence on the effectiveness of telemedicine interventions in diabetes, dyslipidemia, and hypertension: an umbrella review of systematic reviews and meta-analyses. J Med Internet Res 2020;22(3):e16791DOI. 16791PMID:32186516.

https://doi.org/10.2196/

[17] Flocard M, et al. Prise en charge par téléconsultation des patients diabétiques dans le contexte de la pandémie de la COVID-19 : étude prospective observationnelle. Med Mal Metab (2020), 10.1016/j.mmm.2020.11.002

[18] Telemonitoring in diabetes: evolution of concepts and technologies, with a focus on results of the more recent studies ; Emmanuel Andrès, Laurent Meyer, Abrar-Ahmad Zulfiqar,Mohamed Hajjam, Samy Talha,Thibault Bahougne, Sylvie Ervé, Jawad Hajjam,Jean Doucet, Nathalie Jeandidier, and Amir Hajjam El Hassani ; J Med Life. 2019 Jul-Sep; 12(3): 203-214 ; doi: 10.25122/jml-2019-0006

[19] Telemedicine practice guidelines. Accessed March 27, 2020.

[20] Telehealth consultations in general practice during a pandemic lockdown: survey and interviews on patient experiences and preferences ; Fiona Imlach, Eileen McKinlay, Lesley Middleton, Jonathan Kennedy, Megan Pledger, Lynne Russell, Marianna Churchward,Jacqueline Cumming ,Karen McBride-Henry ; BMC Family Practice volume 21, Article number: 269 (2020) 